\title{
Espacios disponibles I.
}

\section{Liminalidad e hibridación lingüística en la escultura de campo expandido}

Available Spaces I: Liminality and linguistic hybridity in expanded field sculpture.

\author{
Jesús MARÍN-CLAVIJo
}

Profesor de escultura y artista plástico. Universidad de Málaga, Málaga, España.

\section{Resumen}

Esta serie fotográfica o fotoensayo es resultado del proyecto de investigación La escultura como interfaz histórico de luz sólida del Plan Propio de la Universidad de Málaga, el cual consiste en la investigación experimental artística basada en el lenguaje escultórico y sus hibridaciones con otras disciplinas, como la fotografía. Para ello he usado herramientas metodológicas como la intervención site-specific, la fotoluminocinética y las posibilidades que la escultura de campo expandido ofrece en cuanto a las narrativas que el objeto significante del entorno escogido puede ofrecer a lo que sucede en él. La fotoluminocinética es una hibridación entre el lenguaje escultórico y el fotográfico, es el corpus teórico de mi tesis principal La fotografía como medio de la escultura en movimiento, y una de mis principales líneas de investigación artística. La experimentación plástica se centra en la intervención fotoluminocinética, o fotografía en exposición prolongada mientras se actúa con elementos luminosos en movimiento, en diversas partes del edificio, arcos de sus fachadas y zonas internas. A través de un diálogo con su arquitectura, con el entorno escogido la obra artística le transfiere significado, aumentándole la carga monumental y estatuaria, y éste a su vez, dota al trabajo artístico de

Ensayo visual

Visual Essay

Correspondencia/ Correspondence Jesús Marín-Clavijo jmarin@uma.es

Financiación/Fundings Sin financiación

Received: 14.09.2020 Accepted: 19.12.2020 narratividad poética.

PALABRAS CLAVE: Fotoluminociética, hibridación, escultura de campo expandido / Photolumino-kinetics, Hybridisation, Expanded field Sculpture.

\section{CÓMO CITAR ESTE TRABAJO / HOW TO CITE THIS PAPER}

Marín-Clavijo, J. (2020). Espacios disponibles I. Liminalidad e hibridación lingüística en la escultura de campo expandido. Umática. Revista sobre Creación y Análisis de la Imagen, 3.

https://doi.org/10.24310/Umatica.2020.v2i3.11189

Umática. 2020; 3:265-285 


\section{El proyecto investigador.}

Esta serie se enmarca en un escenario paradigmático, el Trapiche del Prado, edificio del siglo XVII, bien del patrimonio industrial y cultural ubicado en Marbella, el cual es el resultado de siglos de transformación debidas a su función preindustrial cambiante.

El proyecto investigador se propone varios objetivos, uno de ellos es la reivindicación del edificio dentro del tejido urbano de la ciudad, cobra especial significado gracias a su importancia histórica como a su situación geográfica, en los primeros siglos de su existencia se situaba en el extrarradio del entonces pueblo, y ya desde mediados del siglo XX, completamente integrado en la ahora ciudad de Marbella. Una estructura preindustrial de gran envergadura que va decayendo hasta convertirse en una magnífica ruina, mientras que va siendo engullida por el crecimiento de la trama urbana. Su carácter funcional en tanto que industrial, que se ha ido transformando a lo largo de los siglos de funcionamiento, pasa a ser metafórico y monumental gracias al contraste de la factura arquitectónica en relación al urbanismo en donde se sitúa, su estética derruida contrasta con la novedad arquitectónica del entorno, el cual ha ido rodeando al enclave poco a poco.

Otro de los objetivos fundamentales es la exploración de la capacidad monumental y estatuaria que recupera el hecho escultórico gracias a la recolocación en un lugar emblemático determinado y cómo este entorno también adquiere dimensión histórica y social a partir de la mirada artística. Su identidad totalmente distinta a los edificios residenciales que lo rodean, y también su particular estado ruinoso y abandonado hacen de este conglomerado arquitectónico un enclave que traslada al visitante a otra realidad, al modo en que una puerta o un vano es el mecanismo de transición entre dos ámbitos. Este carácter liminal o fronterizo, umbral entre lugares, se explora mediante la intervención escultórica en los vanos de las arcadas que se encuentran en dos de las fachadas principales del complejo.

\section{Las acciones con luz en movimiento: hibridación de lenguajes}

Continuando con la serie de intervenciones fotoluminocinéticas que desde el año 1997 voy desarrollando, es decir, usando el método de la acción lumínica recogida y documentada mediante la fotografía en exposición prolongada, elaboro esta serie de acciones titulada Espacios disponibles, de la que presento en este foto-ensayo, una primera subserie de 8 piezas.

En ese año comencé la exploración plástica de las posibilidades que ofrece la luz como material escultórico. Su relación con los espacios cotidianos o arquitectónicos, la manera de evidenciarlos gracias a ella y su movimiento, la ocupación de los mismos a través

1. Este concepto es uno de los resultados tangibles de la investigación desarrollada en la Tesis doctoral La captura de la luz en movimiento como medio (Marín-Clavijo, 2015). Es la denominación de la tipología de obra artística que se basa en la exposición prolongada fotográfica y mediante la cual es posible percibir trayectorias y formas espaciales elaboradas mediante el movimiento de elementos luminosos. 
de su traslación y desarrollo en estos volúmenes interiores en los que podemos proyectar nuestra experiencia.

Toda la serie está realizada mediante la acción directa en el espacio dado de las arcadas del Trapiche del Prado, es decir, además de la metodología y lenguaje experimental que se explora, el componente site-specific es fundamental para entender el carácter singular de la obra. El entorno seleccionado, las ruinas de un complejo preindustrial, los restos de un edificio auto excluido del tejido urbano que lo rodea tanto desde un punto de vista espacial como temporal, este se ofrece como un lienzo o escenografía soportante del discurso que se establece en cada frase que se ejecuta.

\section{Liminalidad.}

El término liminalidad, derivado de la palabra latina "limen", que significa "umbral" o "frontera", se refiere a un estado subjetivo psicológico, neurológico o metafísico, consciente de estar en la zona de transición de o entre dos planos existenciales diferentes. Es una noción que abarca la ambigüedad y la indeterminación, y se manifiesta en muchas formas dentro del arte contemporáneo. Es un concepto difícil de describir visualmente, pero ha sido un recurso o elemento narrativo que ha sido usado de diversa manera por artistas contemporáneos como el escultor español Chillida (Lomelí, 2014).

Desde un punto de vista antropológico, este término fue conceptualizado originalmente por primera vez en 1909 por Arnold Van Gennep (1969), a través de sus estudios antropológicos sobre los rituales de transición, de paso de una edad a otra o de un estatus social a otro. Su texto, The Rites of Passage presenta la noción de que el estado liminal acompaña "todo cambio de lugar, estado, posición social y edad" ya que reconoce el "umbral como el comienzo de un estado o acción"

Cincuenta y ocho años después, las teorías de Van Gennep sobre la liminalidad influyeron a su vez en Victor Turner, un antropólogo cultural, que se interesó especialmente en el umbral liminal que existe en un rito de paso entre las "fases de separación y reincorporación"

Turner concibió la liminalidad como un "período de ambigüedad; de estado marginal y de transición". Tanto Turner como Van Gennep exploraron la noción de que todos los ritos de paso se dividen en tres etapas: separación, un período liminal y agregación.

Sin embargo, Turner (1967) afirma que los estados liminales no están necesariamente "confinados a la definición cultural". Así mismo, considera que la vida del hombre se puede considerar que está en permanente crisis, puede producirse en ella cualquier cambio de un estado a otro en cualquier momento. Como resultado, la definición de liminalidad ha evolucionado para incluir la indefinición, ambigüedad, apertura e indeterminación.

Por otro lado, el filósofo español Eugenio Trías estudia ampliamente la idea de límite lle-gando a ser un asunto central en su obra. En títulos como Los límites del Mundo (1986) o La idea de Límite (2006) Trías defiende el rango ontológico del concepto al contrario que Kant, Hegel o el mismo Wittgenstein, que lo consideraban como restrictivo o lógico-lingüístico. 
Gracias a este punto de partida, concluye Trías que pudo definir lo que somos como habitantes del límite o de la frontera entendida en su acepción topológica romana, espacio donde es posible vivir o alimentarse como seres limítrofes.

A partir de la categoría topológica de esta idea, Foucault (1978) elabora su propuesta de Utopías y heterotopías, espacios imposibles y reales pero no considerados como normalizados. En-tiendo sus espacios heterotópicos desde el concepto ya alumbrado por Trías y Turner, lugares habitables para momentos de transición, según Foucault son "espacios otros", "contra-espacios", "lugares reales fuera de todo lugar". Estos son residuos espaciales que las sociedades determina en las áreas que las rodean y que están reservados a aquellos que pueden representar una deriva o desviación a la normalidad exigida que rige la vida cotidiana de esa sociedad. Para Foucault, el paradigma del lugar heterotópico es el cementerio, la ruina, lo abandonado, es el lugar "absolutamente otro".

Así pues, y teniendo esta base conceptual que suporta la categoría de espacio heterotópico y fronterizo, la elección del escenario en el que se realizan las fotoluminocinéticas que aquí se presentan es fundamental para entender la naturaleza de la propuesta plástica. Los espacios, los vanos de las arcadas son el lugar idóneo para que los distintos mensajes cobren singular relevancia. Lugares otros en donde lo fronterizo tiene lugar, lugares habitables para estos ritos de paso que son las obras efímeras que elaboro.

Además de la interdisciplinariedad lingüística, en cuanto a la metodología empleada que se desarrolla en las piezas, la ambigüedad y transitoriedad, lo efímero del material con el que se construyen y su entidad eminentemente transparente al modo en que lo deduce Trías a partir del estudio del Gran vidrio Duchampiano, se genera el verdadero sentido de las obras mediante el reflejo de las mismas en el entorno liminal, tanto por la literalidad de la arquitectura como por la naturaleza del lugar en donde se desarrollan. Es decir, los distintos umbrales de paso, esta arquitectura que sostiene el devenir de lo trazado sutilmente en el intangible aire, son parte consustancial de la pro-puesta ontológica que emerge de la aparición de la palabra en el soporte fotográfico. 


\section{6}

\section{Espacios Disponibles}

ESPACIO ESTÉTICO DISPONIBLE

ESPACIO ESPIRITUAL DISPONIBLE

ESPACIO MORAL DISPONIBLE

ESPACIO FORMAL DISPONIBLE

ESPACIO POLÍTICO DISPONIBLE

ESPACIO SOCIAL DISPONIBLE

ESPACIO POÉTICO DISPONIBLE

ESPACIO DE REVOLUCION DISPONIBLE

ESPACIO REVOLUCIONARIO DISPONIBLE

ESPACIO DE MANSEDUMBRE DISPONIBLE

ESPACIO MANSO DISPONIBLE

ESPACIO DEMOCRÁTICO DISPONIBLE

ESPACIO DICTATORIAL DISPONIBLE 


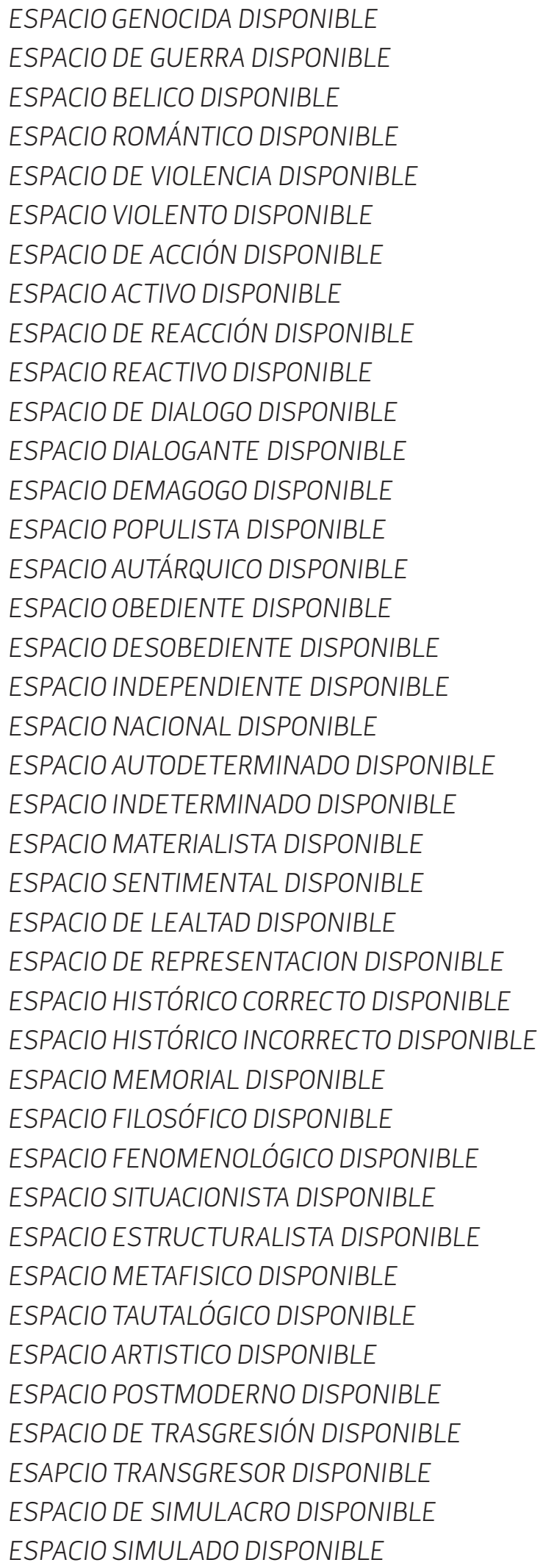


ESPACIO HIPOTÉTICO DISPONIBLE ESPACIO LÓGICO DISPONIBLE ESPACIO UTÓPICO DISPONIBLE ESPACIO IDÉNTICO DISPONIBLE ESPACIO DISTÓPICO DISPONIBLE ESPACIO CAMUFLADO DISPONIBLE ESPACIO INTERRUMPIDO DISPONIBLE ESPACIO DISRUPTIVO DISPONIBLE ESPACIO ANALITICO DISPONIBLE ESPACIO SINTÉTICO DISPONIBLE ESPACIO ESPACIAL DISPONIBLE ESPACIO VIRTUAL DISPONIBLE ESPACIO VIRTUOSO DISPONIBLE ESPACIO REAL DISPONIBLE ESPACIO DISMINUIDO DISPONIBLE ESPACIO ANIMAL DISPONIBLE ESPACIO HUMANO DISPONIBLE ESPACIO MÍNIMO DISPONIBLE ESPACIO VITAL DISPONIBLE ESPACIO SURREAL DISPONIBLE ESPACIO IRREAL DSIPONIBLE ESPACIO DE IDENTIDAD DISPONIBLE ESPACIO IDÉNTICO DISPONIBLE ESPACIO DE PARALISIS DISPONIBLE ESPACIO PARALIZADO DISPONIBLE ESPACIO SEXUAL DISPONIBLE ESPACIO ONANISTA DISPONIBLE ESPACIO MASCULINO DISPONIBLE ESPACIO FEMENINO DISPONIBLE ESPACIO HOMOSEXUAL DISPONIBLE ESPACIO HETEROSEXUAL DISPONIBLE ESPACIO TRANS DISPONIBLE ESPACIO IRREVERENTE DISPONIBLE ESPACIO OPRIMIDO DISPONIBLE ESPACIO REPRIMIDO DISPONIBLE ESPACIO DESVIADO DISPONIBLE ESPACIO PERVERSO DISPONIBLE ESPACIO NARCISISTA DISPONIBLE ESPACIO SELFI DISPONIBLE ESPACIO DE DERRIBO DISPONIBLE 
ESPACIO RESIDUAL DISPONIBLE ESPACIO LIMINAL DISPONIBLE ESPACIO SUBLIMINAL DISPONIBLE ESPACIO RELIGIOSO DISPONIBLE ESPACIO LAICO DISPONIBLE ESPACIO LEGAL DISPONIBLE ESPACIO ILEGAL DISPONIBLE ESPACIO ALEGAL DISPONIBLE ESPACIO CULPABLE DISPONIBLE ESPACIO INOCENTE DISPONIBLE ESPACIO DELINCUENTE DISPONIBLE ESPACIO ASESINO DISPONIBLE ESPACIO HOMICIDA DISPONIBLE ESPACIO VICTIMA DISPONIBLE ESPACIO IRRESPONSABLE DISPONIBLE ESPACIO RESPONSABLE DISPONIBLE ESPACIO DE POSE DISPONIBLE ESPACIO ADOLESCENTE DISPONIBLE ESPACIO ADULTO DISPONIBLE ESPACIO INFANTIL DISPONIBLE ESPACIO VANDÁLICO DISPONIBLE ESPACIO DE PODER DISPONIBLE ESPACIO LEAL DISPONIBLE ESPACIO TRAIDOR DISPONIBLE ESPACIO REBELDE DISPONIBLE ESPACIO PRIVADO DISPONIBLE ESPACIO PÚBLICO DISPONIBLE ESPACIO VERDADERO DISPONIBLE ESPACIO MENTIROSO DISPONIBLE ESPACIO DELICTIVO DISPONIBLE ESPACIO PSICÓTICO DISPONIBLE ESPACIO NERVIOSO DISPONIBLE ESPACIO PSICOPATA DISPONIBLE ESPACIO DE REFERENCIA DISPONIBLE ESPACIO REFERENCIAL DISPONIBLE ESPACIO REFERENTE DISPONIBLE ESPACIO MENTAL DISPONIBLE ESPACIO NATURAL DISPONIBLE ESPACIO ROBADO DISPONIBLE ESPACIO NARRATIVO DISPONIBLE 
ESPACIO LITERAL DISPONIBLE ESPACIO LITERARIO DISPONIBLE ESPACIO DE MONOLOGO DISPONIBLE ESPACIO APARTE DISPONIBLE ESPACIO MITINERO DISPONIBLE ESPACIO DISCURSIVO DISPONIBLE ESPACIO GLOSADO DISPONIBLE ESPACIO DE ARENGA DISPONIBLE ESPACIO DE DISCUSIÓN DISPONIBLE ESPACIO DISPONIBLE DISPONIBLE ESPACIO POTENCIAL DISPONIBLE ESPACIO IMPOTENTE DISPONIBLE ESPACIO SIMULADO DISPONIBLE ESPACIO DISIMULADO DISPONIBLE ESPACIO PROPAGANDÍSTICO DISPONIBLE ESPACIO PERDIDO DISPONIBLE ESPACIO EMOCIONAL DISPONIBLE ESPACIO ALTERNATIVO DISPONIBLE ESPACIO DE MASAJE DISPONIBLE ESPACIO MUELLE DISPONIBLE ESPACIO CÓMODO DISPONIBLE ESPACIO INCÓMODO DISPONIBLE ESPACIO RICO DISPONIBLE ESPACIO POBRE DISPONIBLE ESPACIO DE OFERTA DISPONIBLE ESPACIO AUTOMÁTICO DISPONIBLE ESPACIO NEGRO DISPONIBLE ESPACIO BLANCO DISPONIBLE ESPACIO VERDE DISPONIBLE ESPACIO AZUL DISPONIBLE ESPACIO ROSA DISPONIBLE ESPACIO ROJO DISPONIBLE ESPACIO AMARILLO DISPONIBLE ESPACIO NARANJA DISPONIBLE ESPACIO MORADO DISPONIBLE ESPACIO EQUIDISTANTE DISPONIBLE ESPACIO REVELADO DISPONIBLE ESPACIO RADICAL DISPONIBLE ESPACIO MODERADO DISPONIBLE ESPACIO MENGUANTE DISPONIBLE 
ESPACIO INSULTANTE DISPONIBLE ESPACIO ESTÚPIDO DISPONIBLE ESPACIO DOBLE DISPONIBLE ESPACIO INFINITO DISPONIBLE ESPACIO FINITO DISPONIBLE ESPACIO CUANTICO DISPONIBLE ESPACIO AUTONOMO DISPONIBLE ESPACIO RUIDOSO DISPONIBLE ESPACIO SONORO DISPONIBLE ESPACIO MUSICAL DISPONIBLE ESPACIO DESHAUCIADO DISPONIBLE ESPACIO OKUPADO DISPONIBLE ESPACIO NEGATIVO DISPONIBLE ESPACIO POSITIVO DISPONIBLE ESPACIO CAPRICHOSO DISPONIBLE ESPACIO VISCOSO DISPONIBLE ESPACIO ALUCINANTE DISPONIBLE ESPACIO ABURRIDO DISPONIBLE ESPACIO VULNERABLE DISPONIBLE ESPACIO ANODINO DISPONIBLE ESPACIO INDIFERENTE DISPONIBLE ESPACIO RENOVADO DISPONIBLE ESPACIO SELFIE DISPONIBLE 



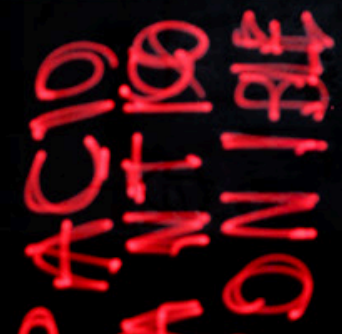

$$
\begin{aligned}
& 0 \leq 0 \text {. } \\
& \text { पू }
\end{aligned}
$$




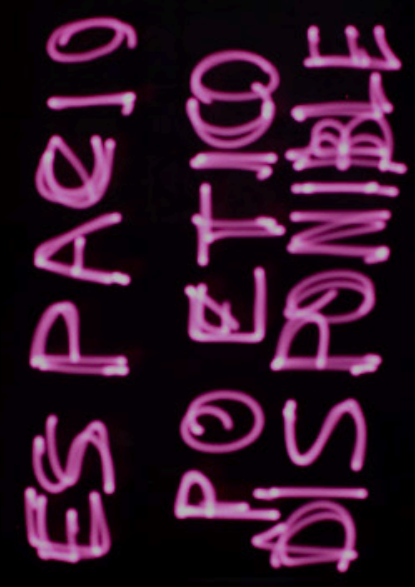




$$
\begin{aligned}
& \text { 웅울 } \\
& \text { 女世 } \\
& \text { A } \underset{\infty}{\stackrel{n}{a}}
\end{aligned}
$$

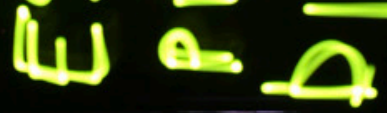




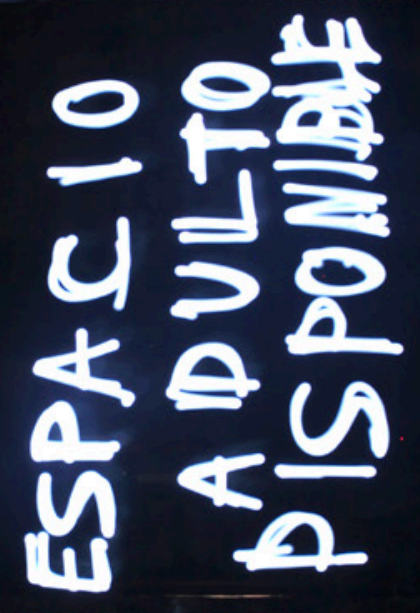




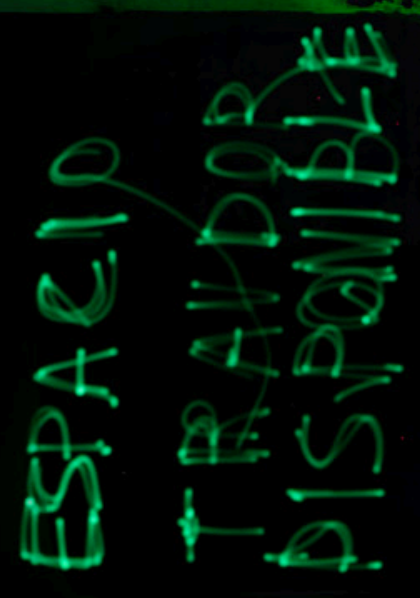




$$
\begin{aligned}
& 0 \text { i) } \\
& \text { 光 } v S \\
& \text { और } \frac{\pi}{\Delta}
\end{aligned}
$$




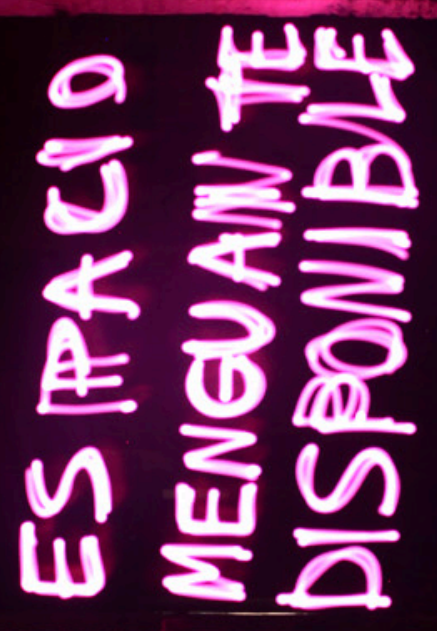




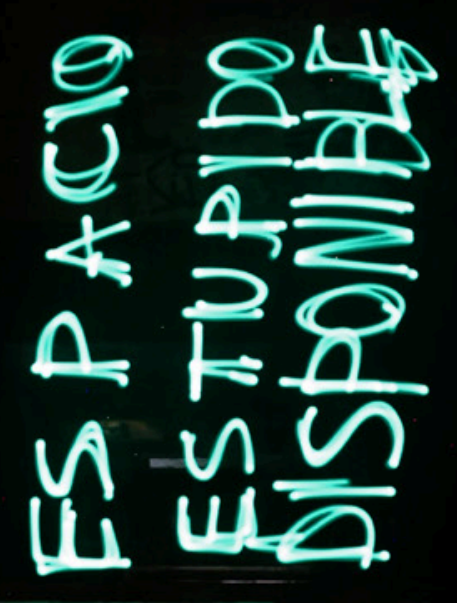




\section{Coda}

El texto poético que acompaña a esta primera subserie 196 Espacios disponibles es el guion narrativo que plantea las distintas posibilidades de fotoluminocinéticas que se desarrollarán en el futuro cercano, muchas de ellas ya elaboradas constituyendo los cuerpos de varias subseries al igual que la que se presenta en este foto-ensayo.

Por otro lado, la hibridación de lenguajes, la estructura formal de las piezas presentadas, además de ser una continuación de una larga trayectoria en mi investigación plástica, se apoya en innumerables trabajos y autores siendo los más notables y más cercanos, teniendo en cuenta la poética del discurso, Vicky DaSilva (Cascone, 2014), Tokihiro Sato (Takuo, 2000) o Jacques Pugin (Girardin, 2020).

Las ideas y conceptos antropológicos y filosóficos que sostienen el corpus teórico de esta propuesta son un fértil campo en el que me apoyo en este proyecto, aunque servirán para posteriores investigaciones, experimentaciones y proyectos de creación en el futuro.

\section{Referencias / References}

MARÍN-CLAVIJO, J., et al. (2015) La captura de la luz en movimiento como medio escultórico [en línea]. Universidad de Málaga. Disponible en: <http://hdl.handle.net/10630/10501> [Consulta: 1 de julio de 2020].

LOMELí PONCE, J. (2014). El límite como concepto plástico en la obra de Eduardo Chillida. Una lectura desde la filosofía del límite de Eugenio Trías. En-claves del pensamiento, 8(15), 13-37.

VAn Gennep, A. (1969). The Rítes of Passage. Chicago: The University of Chicago Press.

TURNER, V. (1967). The Forest of Symbols, Nueva York: Ithaca [traducción castellana: La selva de los símbolos], Siglo XXI, Madrid, 1980.

TRÍAS, E. (1986). Los límites del mundo. El Ciervo, 35(423), 39-39.

TRÍAS, E. (2006). La idea de límite. En La interpretación del mundo: cuestiones para el tercer milenio (pp. 169-190). Anthropos.

FOUCAULT, M. (1978). Espacios otros: utopías y heterotopías. Carrer de la Ciutat, 1978, núm. 1.

CASCONE, S. (2014). Artist Renders East River Flows in Light Graffiti. Artnet [en linea]. Ref. 1 de agosto de 2014. Disponible en: <http://news.artnet.com/in-brief/artist-rendersemeast-river-flowsem-in-lightgraffiti-70748> [Consulta: 27/01/2021].

TAKUO, K. (2000). Breathing Light: The World of Tokihiro Sato. Photoarts [en linea]. Disponible en: <http://photoarts.com/gallery/SATO/satoexh.html> [Consulta:27/01/2021].

GIRARDIN, D. (2020). Jacques Pugin: nota biográfica. Jacques Pugin [en linea]. Disponible en: <http://www.jacquespugin.ch/detailbio?id=152> [Consulta: 27/01/2021]. 